\title{
A BRIEF NOTE ON PSR 1848+04
}

\author{
VALENTÍN BORIAKOFF \\ Worcester Polytechnic Institute \\ ROSSOLINO BUCCHERI \\ IFCAI-CNR \\ Francisco Fauci \\ Istituto di Fisica dell'Universita
}

\begin{abstract}
Average pulse profiles at three frequencies are presented for PSR 1848+04. A highly structured pulse profile is seen (three-component main pulse, complex interpulse), with the longitudinal position of the pulse components invariant with frequency.
\end{abstract}

\section{Observations}

?SR $1848+04,(P=0.284697 \mathrm{~s})$ was discovered durng a search of COS-B $\gamma$-ray source error boxes at he Arecibo Observatory. It has a very complex rofile as shown in figure 1. Detailed characteristics tre given in Boriakoff, Buccheri and Fauci (1984), lowever, the period derivative is smaller than that juoted therein $\left(\dot{P}<0.16 \times 10^{-17} \mathrm{~s} / \mathrm{s}\right)$.

\section{Discussion}

The pulse profile shows four clearly defined comporents, a triple main pulse and an interpulse. The nain pulse central component has a steeper spec;ral index than the main side pulses by $0.39 \pm 0.05$ setween 1414 and $430 \mathrm{MHz}$ and the interpulse spec;ral index is steeper by $0.75 \pm 0.3$ over the same frejuency range. This suggests two emission mechalisms: one for the outer components of the main sulse and another for the interpulse and the main sulse center component. The interpulse may have imission components at both sides of it, most slearly seen at the lower frequencies. These components persist over integrations of different data iets.

We interpret the three-component pulse profile n terms of Rankin's core-cone mechanism (Rankin 1983a). It is generally assumed that the external somponents of the main pulse mark the longitude imits of the emission of the pulsar. In addition, the existence of an interpulse suggests that we see the two opposite poles of the neutron star magnetic ield as it rotates. Perhaps the interpulse side components mirror image the side components of the main pulse. However, most interpulsars have a very small duty cycle (e.g. PSR $0823+26)$, as compared with PSR 1848+04, where emission is observed over

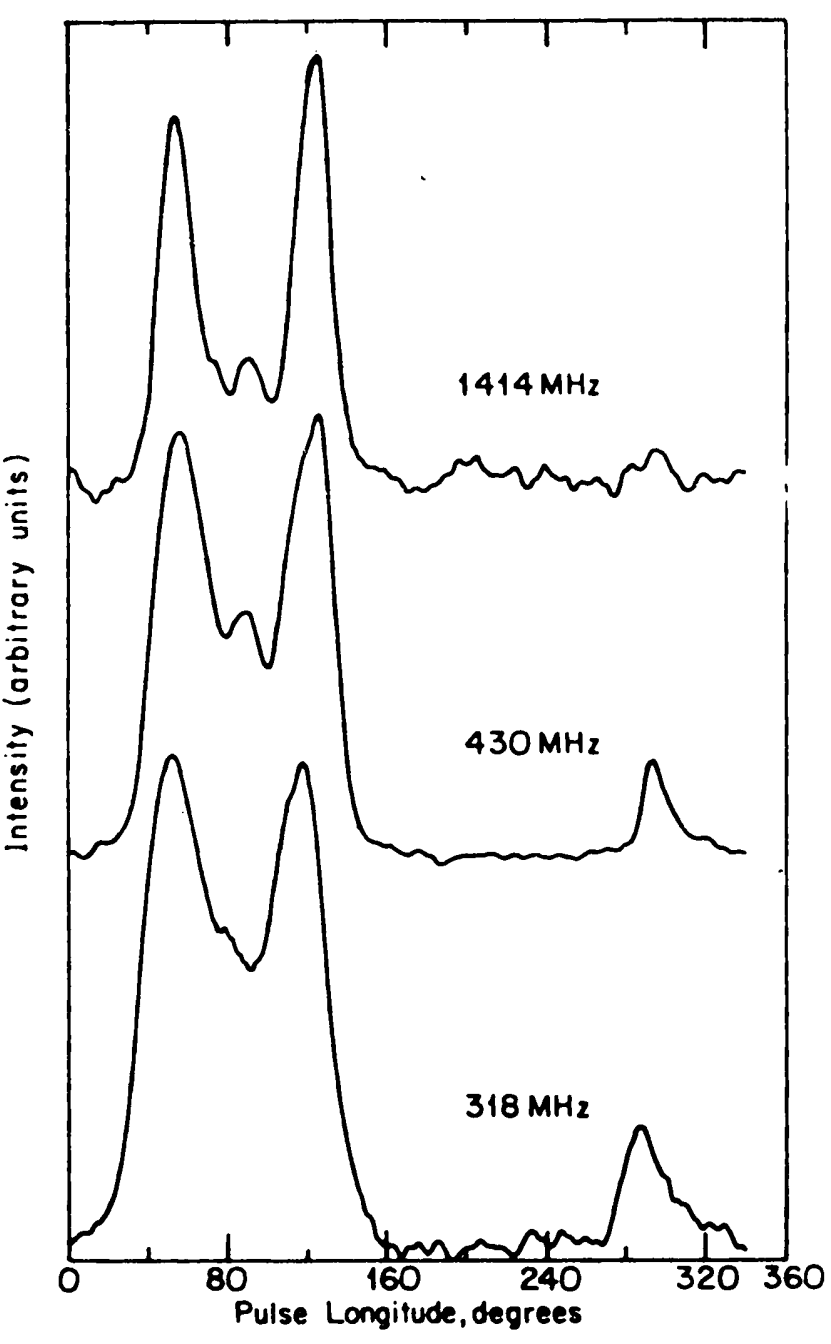

Figure 1 PSR $1848+08$ average pulse profile at $1414 \mathrm{MHz}$ (total intensity), $430 \mathrm{MHz}$ (total intensity) and $318 \mathrm{MHz}$ (single linear polarization) aligned at the main pulse center point. $94 \%$ of the period is shown.

$180^{\circ}$ longitude. In view of the large duty cycle an alternative interpretation is that, as in the case of PSR 0950+08 (Hankins and Cordes 1981), the rota- 
tion axis is nearly aligned with the line of sight, so we see only one polar cap during a rotation period. However, in such cases a regular three component main pulse profile is not expected. Another noteworthy feature is that the separation between the peaks of the side components of the main pulse does not change with frequency, at $318 \mathrm{MHz}$ it is in fact slightly less than at 430 or $1414 \mathrm{MHz}$. This could be the result of polarization differences between the total intensity of $430 \mathrm{MHz}$ and the linear polarization of $318 \mathrm{MHz}$. The location of the interpulse also seems invariant with frequency.

\section{Conclusions}

PSR 1848+04 is a pulsar challenging standard interpretations of magnetospheric structures and it may provide some clues to their organization. Complex pulse profiles are common in pulsars (Rankin 1983a), however, PSR $1848+04$ is of special interest because of the regularity of the components and the behavior of their features.

Acknowledgment: This work has been supported in part by the Arecibo Observatory. The Arecibo Observatory is part of the National Astronomy and Ionosphere Center, which is operated by Cornell University under a management agreement with the National Science Foundation. 